\title{
Índice de Masa Corporal, Velocidad de Marcha y Fuerza de Prensión Manual en Mujeres Mayores Chilenas \\ Body Mass Index, Gait Speed and Manual Pressure Force in Chilean Older \\ Women
}

*, **Felipe Hermosilla Palma, ***Luis Felipe Castelli de Campos, ****Marco Cossio Bolaños, *****Cristian Luarte Rocha, ******Grimanés Medina Monsalve, ******Cenaida Garrido Bastías, ******Danny de la Hoz Riquelme, ******Mauricio García Ortega

*Universidad Autónoma de Chile (Chile); **Universidad Adventista de Chile (Chile), ***Universidad del BíoBío (Chile), ****Universidad Católica de Maule (Chile), *****Universidad San Sebastián (Chile), ******Universidad Pedro de Valdivia (Chile)

Resumen. Objetivo: El objetivo del presente estudio es de comparar la velocidad de marcha (VM), fuerza de tren superior (PM) y el Índice de Masa Corporal (IMC) en mujeres mayores que participan de forma regular en talleres de actividad física, según rango de edad, así como, verificar la asociación entre Edad, IMC y PM conVM en 10 metros. Metodología: Participaron voluntariamente 85 mujeres (70,1 $\pm 6,9$ años) vinculadas al programa de talleres de adulto mayor del IND de la región de Nuble. Las participantes fueron categorizadas según rango de edad (grupo total [GT], 60-64, 65-69, 70-74, 75-79 y 80-84 años). Se evaluó el peso corporal y la estatura para el cálculo de IMC, la fuerza de tren superior con la prueba de prensión manual (PM) en la mano dominante y la VM en 10 metros. Resultados: Los principales resultados apuntan que la VM fue significativamente inferior para el grupo de 80-84 años comparados a los grupos GT (p<0.03), 60-64 (p<0.00) y de 65-69 años ( $\mathrm{p}<0.03)$. El grupo 70-74 años fueron significativamente más lento que el de 60-64 años $(\mathrm{p}<0.02)$. La relación entre VM y PM para el grupo analizado fue positiva, baja y no significativa $(r=0,20, p<0.06)$. Por fin, la Edad y el IMC resultaron en un modelo estadísticamente significativo $\left[\mathrm{F}(2,82)=13,784 ; \mathrm{p}<0.001 ; \mathrm{R}^{2}=0.252, \mathrm{EE}=0,204 \mathrm{~m} / \mathrm{s}\right]$ para predicción de laVM. Conclusión: En general, concluimos que la VM se reduce en grupos con edades mayores y que la PM no presenta la misma tendencia, además que la Edad y el IMC son predictores de VM en mujeres mayores.

Palabras clave: adulto mayor, velocidad de marcha, fuerza muscular, índice de masa corporal.

Abstract. The aim of this study is to compare the gait speed (VM), manual pressure force (PM) and the Body Mass Index (IMC) in older women who participate regularly in physical activity programs, according to age, as well as, to verify the association between Age, IMC and PM with MV in 10 meters. 85 older women (70.1 \pm 6.9 years) from the physical activity program of the National Sports Institute of the Nuble / Chile region voluntarily participated in the study. The participants were categorized according to age range (total group [GT], 60-64, 65-69, 70-74, 75-79 and 80-84 years). Body weight and height were evaluated for the calculation of IMC, upper body strength with the manual grip test (PM) in the dominant hand and the MV in 10 meters. Results: The main results indicate that MV was significantly lower for the 80-84 year-old group compared to the GT ( $<<0.03), 60-64$ ( $<<0.00)$ and 65-69 years $(\mathrm{p}<0.03)$. The 70-74 year group were significantly slower than the 60-64 year group $(\mathrm{p}<.024)$. The relationship between MV and PM for the analyzed group was positive, low and not significant $(r=0.20, p<0.06)$. Finally, Age and BMI resulted in a statistically significant model $[\mathrm{F}(2,82)=13,784 ; \mathrm{p}<0.001$; $\mathrm{R} 2=0.252, \mathrm{SE}=0.204 \mathrm{~m} / \mathrm{s}$ ] for prediction of MV. Conclusion: In general, we conclude that MV decreased in older age groups and that PM does not present the same trend and that Age and IMC are predictors of MV in older women.

Keywords: Elderly, gait speed, muscular strength, body mass index.

\section{Introducción}

El vertiginoso incremento de la población de adultos mayores a nivel mundial implica asumir estrategias que permitan favorecer la calidad de vida de los indivi-

Fecha recepción: 25-04-21. Fecha de aceptación: 25-06-21 Felipe Andrés Hermosilla Palma

fhermosilla.pf@gmail.com duos en esta etapa de su ciclo vital. Es así como de acuerdo con datos estimativos de la Organización Mundial de la Salud (OMS), el número de adultos mayores (AM) en el mundo se incrementará de un $11 \%$ a un $22 \%$ entre 2000 y 2050 pasando de 605 millones a 2000 millones de personas en este periodo de tiempo (OMS, 2015). La realidad en Chile no escapa a esta tendencia, proyectándose que un 33\% de la población superará los 60 años y de ésta un 8,9\% alcanzará los 79 años (Arnold, 
Herrera, Massad, \& Thumala, 2018).

Dentro de este contexto, la disminución de la función física asociada al envejecimiento y la inactividad física (IF), ha sido ampliamente descrita en la literatura (Alcañiz \& González-Moro, 2020; Bowden et al., 2019; Kendrick et al., 2014; Valenzuela et al., 2019). Sus implicancias abarcan desde el incremento en el riesgo de caída (Lavedán Santamaría, Jürschik Giménez, Botigué Satorra, Nuin Orrio, \&Viladrosa Montoy, 2015) hasta la pérdida de independencia (Villalobos, 2019), deterioro cognitivo (Poblete-Valderrama et al., 2019), enfermedades metabólicas (Pozo-Cruz et al., 2013), además de un fuerte encarecimiento en los gastos en salud de los gobiernos (OMS, 2018).

Dentro de las estrategias prescritas para atenuar la inminente aparición de estas inhabilidades, el ejercicio físico (EF) ha demostrado ser una estrategia útil y efectiva para conseguir este propósito (Mendonca et al., 2016; Sherrington et al., 2019; Villarreal-Angeles, Moncada-Jimenez, \& Ruiz-Juan, 2020). Es así como mejoras en la condición muscular, uno de los principales factores protectores en la aparición del síndrome de fragilidad, característico en el AM (De Labra, Guimaraes-Pinheiro, Maseda, Lorenzo, \& MillánCalenti, 2015), se advierten a partir de la ejecución de diversos tipos de programas de ejercicios, siendo el entrenamiento multicomponente uno de los más efectivos (Casas-Herrero et al., 2019; Fragala et al., 2019; Jadczak, Makwana, Luscombe-Marsh, Visvanathan, \& Schultz, 2018).

En consecuencia, la evaluación de la condición funcional de los AM, la identificación de variables asociadas a la locomoción, así como de la fuerza muscular, permiten determinar el riesgo de caída y discapacidad en esta población. De este modo, la valoración de la velocidad de marcha y la fuerza de prensión manual se describen como medios idóneos para estimar el riesgo de evento adverso, funcionalidad, independencia/dependencia en AM que viven en la comunidad (Guralnik et al., 2000; Heiland et al., 2016; Mancilla S, Ramos F, \& Morales B, 2016; Rijk, Roos, Deckx, Van den Akker, \& Buntinx, 2016).

Diversos estudios han demostrado que con el transcurso de la edad se va deteriorando la funcionalidad, condición física, calidad de vida, cognición, lo que perjudica su estado de salud (Drey et al., 2016; Siemonsma et al., 2018; Van Houwelingen et al., 2014). Por lo tanto, estudiar el IMC, VM y FPM de las mujeres adultas desde los 60 hasta los 84 años, podría ayudar a identificar el rango de edad donde se produce disminución y/o incremento significativo, así como es posible que exista asociación entre las variables mencionadas.

Así, el objetivo del presente estudio es de comparar y establecer asociaciones entre la velocidad de marcha en 10 metros, fuerza de presión manual y el IMC en mujeres mayores según rango de edad que participan de forma regular en programas de actividades físicas.

\section{Metodología}

Fueron evaluadas 85 mujeres mayores categorizadas según rango de edad, bajo un tipo de muestreo no probabilístico por conveniencia, (grupo total [GT], 60$64,65-69,70-74,75-79$ y 80-84 años) pertenecientes a talleres deportivos del Instituto Nacional del Deporte en la región de Nuble, Chile.

Como criterio de inclusión se consideró las participantes que participan del programa como mínimo de 6 meses, con frecuencia superior a los $90 \%$ de las clases que fueron realizadas, mientras que fueron excluidos del estudio quienes presentaban enfermedades asociadas que podrían comprometer el desempeño en las pruebas físicas realizadas y aquellas que estuviesen fuera del rango entre 60-84 años.

Los talleres fueron realizados durante 9 meses con una frecuencia de dos sesiones semanales, con una duración de 60 minutos cada una. Las actividades ejecutadas se orientan principalmente a una metodología multicomponente, en lo cual se focaliza al desarrollo de la fuerza, resistencia cardio-respiratoria y estabilidad, mediante ejercicios en circuito con peso corporal, bandas elásticas y pesos añadidos, balance dinámico y estático y al estímulo de los componentes coordinativos mediante secuencias coreográficas.

Las participantes fueron informadas y firmaron voluntariamente un consentimiento para poder ser parte de esta. Todos los procedimientos se ajustaron a lo descrito en la declaración de Helsinki para seres humanos.

\section{Procedimiento}

Las evaluaciones fueron realizadas en una cancha indoor para evitar interferencias externas. Los protocolos se realizaron de acuerdo con detalle que se describe a continuación: las evaluaciones se efectuaron durante el horario de realización de las clases, el cual varió dependiendo de acuerdo con la ubicación geográfica del grupo evaluado. La secuencia de testeo comenzó con antropometría para luego evaluar la condición física. Todos los procedimientos estuvieron a cargo de profesionales con amplia experiencia en evaluaciones antropométricas 
y físicas

Para la determinación de las variables antropométricas, se utilizó de mediciones según el protocolo estandarizado propuesto por la Sociedad Internacional para el Avance de la Cineantropometría (ISAK)(Norton \& Olds, 1996). La masa corporal (kg) se estimó con el participante vestido con ropa liviana y descalzo sobre balanza calibrada digital (Tanita, modelo SC 240-MA). La talla (m) se estimó con el participante descalzo utilizando un estadiómetro portable (Seca, modelo 213). Se consideró el índice de masa corporal (IMC) utilizando la fórmula: $\left[\mathrm{kg} / \mathrm{m}^{2}\right]$.

En los aspectos de condición física se evaluó la velocidad de marcha (VM) por medio del tiempo empleado en recorrer en una distancia de 10 metros (Adell, Wehmhörner, \& Rydwik, 2013) con Fotocélulas (Software Chronojump ${ }^{\circledR}$ 1.9.0., Spain), estableciéndose como puntos de corte valores inferiores $1 \mathrm{~m} / \mathrm{seg}$ (Rydwik, Bergland, Forsén, \& Frändin, 2012), los que clasifican a los sujetos con riesgo incrementado de caída. Los sujetos debían recorrer esta distancia a ritmo de caminata habitual y partiendo desde posición estática (Studenski et al., 2011).

La Fuerza de prensión manual se evaluó por medio de un dinamómetro digital marca Jamar ${ }^{\circledR}$ (Roberts et al., 2014). La persona evaluada se ubicó en posición sedente en una silla con el antebrazo apoyado y el codo en flexión de $90^{\circ}$, en esta posición debía aplicar la mayor fuerza que le fuese posible durante 3 segundos, con una pausa de 15 segundos entre repeticiones, teniendo dos intentos por cada mano, registrándose el mejor de ellos por cada miembro (Mathiowetz, 2002). Para la clasificación de los resultados se utilizaron los valores propuestos en el Consenso para el Diagnóstico y Definición de Sarcopenia (Cruz-Jentoft et al., 2019), en donde se establece que registros igual o inferiores a $16 \mathrm{~kg}$ en mujeres son indicadores de disminución de la capacidad física.

\section{Análisis estadístico}

Para efectos estadísticos se aplicó el Test ShapiroWilk $(n<50)$ y Kolmorogov-Smirnov $(n>50)$ para verificar normalidad de los datos y el test de levene para homocedasticidad. Los datos fueron presentados en media, desviación estándar e IC95\%. Se utilizó el test ANOVA one-way y la prueba posthoc de bonferroni para comparación de las medias entre los grupos según el rango de edades. Se utilizó el test de correlación de Pearson y se utilizó la siguiente escala de magnitudes para evaluar los coeficientes de correlación: <.20, muy baja; .21 -.40, baja; <.41-.60, moderado; <.61-.80, fuerte $<$.81-.99, muy fuerte y 1.00, perfecta (Alzina, 1987). Por fin, se realizó la análisis de regresión linear múltiple entre las variables edad y IMC con las variables PM y Ve de forma general y según rango de edad. Todos los procedimientos estadísticos fueron realizados en el GraphPad Prism 8.2 y el nivel de significancia adoptado fue de $\mathrm{p}<0.05$.

\section{Resultados}

En la tabla 1 son presentadas las características de la muestra del estudio. Se observó diferencia estadísticamente significativa $(\mathrm{p}<0.00)$ para la edad entre los grupos analizados y que no hubo diferencia estadísticamente significativa $(\mathrm{p}<0.05)$ entre las variables antropométricas según rango de edad.

Tabla 1 .

Caracterización de la Edad y variables antropométricas de las mujeres mayores estudiadas según el rango de edad. Valores presentados en media y desviación estándar.

\begin{tabular}{|c|c|c|c|c|}
\hline Rango Edad & $\begin{array}{l}\text { Edad } \\
\text { (años) }\end{array}$ & $\begin{array}{c}\text { Peso Corporal } \\
(\mathrm{Kg})\end{array}$ & $\begin{array}{l}\text { Talla } \\
\text { (m) }\end{array}$ & $\begin{array}{c}\mathrm{IMC} \\
\left(\mathrm{Kg} / \mathrm{m}^{2}\right)\end{array}$ \\
\hline GT $(n=85)$ & $70,1 \pm 6,9$ & $67,6 \pm 10,8$ & $1,55 \pm 0,07$ & $28,4 \pm 5,2$ \\
\hline $60-64(n=19)$ & $61,5 \pm 1,5^{a}$ & $66,8 \pm 10,2$ & $1,56 \pm 0,10$ & $27,8 \pm 4,7$ \\
\hline $65-69(\mathrm{n}=23)$ & $66,8 \pm 1,3^{b}$ & $70,3 \pm 13,8$ & $1,52 \pm 0,08$ & $30,7 \pm 7,2$ \\
\hline $70-74(\mathrm{n}=20)$ & $71,7 \pm 1,2^{\mathrm{b}, \mathrm{c}}$ & $66,1 \pm 8,9$ & $1,56 \pm 0,05$ & $27,4 \pm 4,3$ \\
\hline $75-79(\mathrm{n}=11)$ & $76,5 \pm 1,7^{a, b, c}$ & $66,4 \pm 12,1$ & $1,58 \pm 0,05$ & $26,7 \pm 4,3$ \\
\hline $80-84(\mathrm{n}=12)$ & $81,7 \pm 1,6^{a, b, c, d}$ & $67,3 \pm 6,7$ & $1,55 \pm 0,04$ & $27,9 \pm 2,3$ \\
\hline ANOVA p-value & 0.001 & 0.83 & 0.32 & 0.23 \\
\hline
\end{tabular}

La Velocidad de la Marcha en la caminata de 10 metros (VM) y la fuerza de Prensión Manual (PM) fueron comparadas según los rangos de edad en la figura 1. Se observó que los valores de VM y PM para el grupo total (GT) presentaron promedio de 1,28 $\pm 0,23 \mathrm{~m} / \mathrm{s}$ y $20,3 \pm 3,9 \mathrm{Kg}$, respectivamente. Se observó solamente diferencia estadísticamente significativa en la VM $(\mathrm{p}<0.00)$ entre los grupos analizados. El grupo de 8084 años fueron significativamente más lentos que el GT, de 60-64 años y que el de 65-69 años. También se observó que los grupos de 70-74 años fueron significativamente más lento que el de 60-64 años.
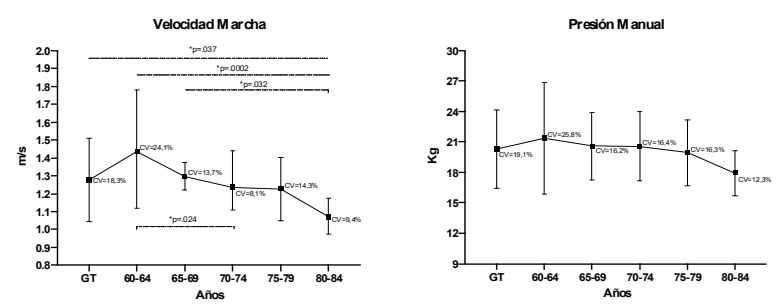

Figura 1. Resultados de la velocidad de Marcha y de la Presión Manual de las mujeres mayores según el rango de edad. Valores presentados en media y desviación estándar.

La relación entre VM y PM para las mujeres mayores fue positiva, baja y no significativa $(r=0.20)$ y al 
utilizar el análisis de regresión linear simples se observa que la $\mathrm{PM}$ no presenta asociación estadísticamente significativa con la VM $\left[F(1,83)=3,399, p=0.06 ; R^{2}=\right.$ 0.039].

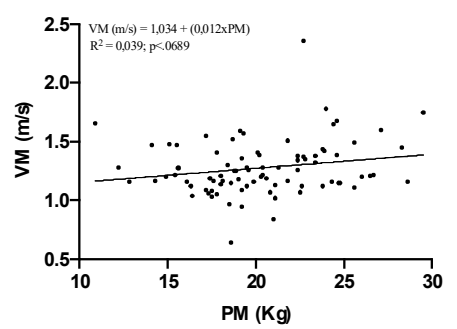

Figura 2. Relación entre velocidad de Marcha y Presión Manual de las mujeres mayores

Finalmente, se realizó la regresión linear múltiple para verificar si la Edad y el IMC pueden prever la VM en mujeres mayores. La análisis resultó en un modelo estadísticamente significativo $[\mathrm{F}(2,82)=13,784 ; \mathrm{p}<0.00$; $\left.\mathrm{R}^{2}=0.252\right]$ considerando la Edad $(\hat{\boldsymbol{a}}=-0,484 ; \mathrm{t}=-5,043$; $\mathrm{p}<0.00)$ y el IMC $(\hat{\boldsymbol{a}}=-0,184 ; \mathrm{t}=-1,92 ; \mathrm{p}<0.04)$ como previsores de la VM.

Tabla 2.

Resultados del análisis de regresión linear múltiple para la predicción de la velocidad de la marcha en la prueba de caminata de 10 metros em mujeres mayores.

\begin{tabular}{cccccc}
\hline \multirow{2}{*}{ Variables } & \multirow{2}{*}{ Coeficiente $\beta$} & \multirow{2}{*}{ EE } & \multicolumn{2}{c}{ IC95\% } & \multirow{2}{*}{ p-value } \\
\cline { 4 - 5 } & & & Mín & Máx & \\
\hline Constante & 2,669 & 0,271 & 2,130 & 3,207 & $<0.000$ \\
Edad (años) & $-0,484$ & 0,003 & $-0,023$ & $-0,010$ & $<0.000$ \\
IMC $\left(\mathrm{kg} / \mathrm{m}^{2}\right)$ & $-0,184$ & 0,004 & $-0,017$ & 0,000 & $<0.000$ \\
\hline
\end{tabular}

$\mathrm{VM}(\mathrm{m} / \mathrm{s})=2,669-(0,016 \times \mathrm{Edad})-(0,008 \times \mathrm{IMC}) ; \mathrm{R}^{2}=0,252$ (error estándar de la estimativa $=0,204 \mathrm{~m} / \mathrm{s})$. EP - Erro padrão; IC - intervalo de confianza

\section{Discusión}

El objetivo del presente estudio fue comparar a velocidad de marcha, fuerza de tren superior y el IMC en mujeres mayores que participan de forma regular en actividades físicas, según rango de edad y verificar la asociación entre Edad y IMC con variables de fuerza de presión manual (PM) y Velocidad de marcha (VM). Los principales resultados observados en el presente estudio es la evidencia de una pérdida significativa de fuerza en miembros inferiores, determinada a partir de los descensos en la VM, entre los grupos de edad analizados, siendo el grupo de mayor edad (80-84 años) el que menores índices presenta. Esta situación no ocurre con la fuerza de miembros superiores medidos por medio de la PM, no observándose diferencias estadísticamente significativas entre los distintos rangos de edad analizados, a pesar de observar una tendencia de disminución de los valores promedios en función de la edad. Esa diferenciación puede estar condicionada de que la capaci- dad contráctil de musculatura, principalmente el realizar una secuencia de contracciones musculares como es en el test de VM puede verse más afectada en función de la edad de lo que la producción de fuerza en una única contracción como es el test de PM, así como, la mayor cantidad de fuerza muscular exigida para la realización de determinada tarea puede generar mejores indicativos del estado global del individuo, aspecto que puede ser refrendado por los diagnósticos de sarcopenia, en donde la identificación del estado de severidad en el deterioro de la función se basa en pruebas de desplazamiento y equilibrio, como Timed Up and Go, caminata en 400 mts., velocidad de marcha y SPPB (Cruz-Jentoft et al., 2019).

En concordancia con nuestros hallazgos, (Yang et al., 2020), observaron que los grupos con mayor edad también presentaron valores inferiores en la VM $(\mathrm{p}<0.00)$ $y$, por otro lado, verificaron reducción significativa en la capacidad de producir fuerza (PM) en grupos de mayor edad $(\mathrm{p}<0.00)$.

La VM es considerada una herramienta clínica simple y accesible para evaluar la salud de la población mayor (Rydwik et al., 2012). Studenski et al., (2011), quienes analizaron 9 estudios de cohorte, en donde se buscó identificar la relación entre la velocidad de marcha y la supervivencia en un periodo de 5 años. Los resultados indican que la velocidad de marcha va disminuyendo en función de la edad, así como la supervivencia, demostrando que el presente test puede ser indicativo de longevidad también.

Por otro lado, nuestros resultados indican una baja correlación y no significativa entre PM y VM, medido en una distancia de 10 metros, indicando que la fuerza máxima isométrica de tren superior, medida por medio de la prensión manual no fue capaz de presentar una tendencia linear con laVM. Tal condición puede asociarse a que las pruebas exigen diferentes manifestaciones de la fuerza y, también la modelación de las actividades que desarrollan. Los mismos hallazgos son reportados por Muehlbauer et al., (2012) quienes investigaron respecto de la relación entre las variables de fuerza, potencia y equilibrio en 74 adultos mayores sanos y físicamente activos (edad 70 \pm 5 años). La velocidad de marcha, evaluada con el test de caminata de $10 \mathrm{mts}$., fue una de las variables identificadas para determinar el equilibrio dinámico, encontrándose correlaciones no significativas entre ésta y la fuerza isométrica para miembros inferiores. Por otro lado, estudios reportan que la fuerza de agarre es un buen predictor de pérdida de movilidad comparado con los test de velocidad de mar- 
cha asociados a cambios de dirección como Timed up and go (TUG) $(\mathrm{p}<0.00)$ (Garber et al., 2011). Del mismo modo, Rijk et al., (2016) en su meta-análisis, concluyen que la PM presenta validez predictiva para pérdida de movilidad, funcionalidad y mortalidad. De acuerdo con esto, se observa la necesidad de estudios más conclusivos de la relación entre VM y PM, principalmente por los diferentes protocolos existentes en la literatura de ambos los test, lo que dificulta estudios comparativos.

En general, las evidencias científicas reportan un detrimento de la condición funcional de los adultos a medida que envejecen, o sea, presentan reducción del estado de acondicionamiento físico y de la capacidad para la realización actividades de la vida diaria y que el ejercicio físico ha demostrado ser una estrategia eficaz para atenuar esta condición (Mendonca et al., 2016), por lo que el control y evaluación de la condición es factor fundamental para la compresión de los beneficios de los programas de actividad física. Partiendo de ese concepto, estudios han presentado resultados considerados como punto de corte para el desempeño en la VM y PM lo que puede representar situación contextualizada de aumentado para riesgo de caídas y de la condición física global, respectivamente (Chou et al., 2019; Cruz-Jentoft et al., 2019; Kuki et al., 2019; Sallinen et al., 2010). Sumado a eso, las recomendaciones de actividad física son de 150 minutos semanales en AF con intensidad moderada o 75 minutos con intensidad vigorosa (Garber et al., 2011). En el presente estudio, como limitación no se realizó el control de la intensidad de las actividades realizadas, pero se observó que todos los grupos presentaron valores promedios superiores a los reportados, lo que indican que, en la población de mujeres mayores que practican actividad física direccionada a un trabajo multicomponente de 2 veces a la semana con duración de 60 minutos en cada sesión son satisfactorios para la mantención o mejora de la condición física y en especial, impactando directamente en la reducción de los riesgos de caídas, una de las principales preocupaciones en adultos mayores. En relación con la duración de las intervenciones Bellomo et al., (2013). desarrollaron un protocolo de entrenamiento sensoriomotor, contrarresistencia y de electroestimulación en adultos mayores durante 12 semanas. Los grupos de entrenamiento sensorio motor y contrarresistencia fueron intervenidos dos veces por semana. La sesión del grupo sensoriomotor tuvo una duración inferior a 60 minutos (10 minutos de calentamiento - 20 minutos de intervención sensoriomotora). Los efectos del entrenamien- to fueron determinados mediante dinamometría para miembros inferiores y estabilidad en prueba de caminata, reportándose diferencias estadísticamente significativas para fuerza $(\mathrm{p}<0.01$ en el grupo sensoriomotor; $\mathrm{p}<0.05$ en grupo contrarresistencia) así como en la longitud del paso y el tiempo de contacto $(\mathrm{p}<0.00$ en el grupo sensoriomotor; $\mathrm{p}<0.05$ en grupo contrarresistencia)

El propósito de comprender la asociación de las variables antropométricas, fuerza de tren superior y la edad con la VM, se observó en nuestro estudio que la Edad y el IMC resultaron en un modelo estadísticamente significativo como previsores de la VM. En ese mismo sentido, Fragala et al., (2012) reportaron asociaciones positivas similares a nuestros hallazgos $\left(\mathrm{R}^{2}=0.229\right.$ $\mathrm{p}<0.00$ para Edad vsVM), concluyendo que la edad puede ser un factor determinante para el rendimiento funcional en mujeres mayores. Del mismo modo, Novaes, Miranda, \& Dourado, (2011) encontraron asociación estadísticamente significativas entre Edad y VM.

Los principales resultados obtenidos de la investigación han demostrado que el valor del IMC para todos los grupos fue superior a los valores ideales indicados por la OMS. Además de esto, no se encontraron Correlaciones entre esta variable y el riesgo de caída determinado a través de la velocidad de desplazamiento. Gonzalez, Gates, \& Rosenblatt, (2020) compararon variables asociadas a la marcha en un grupo de adultos mayores normopeso y obesos $(n=37)$, encontrando diferencias estadísticamente significativas $(p<0.05)$ entre ambos, en velocidad de marcha en $10 \mathrm{mts}$ yTimed Up and Go en favor del grupo normopeso. Si bien es cierto los autores reportan correlaciones débiles y significativas entre la estabilidad al caminar y el IMC, concluyen que estas no son indicativas de aumento del riesgo de caída en este grupo. Del mismo modo, la prensión manual no correlacionó con el IMC. Sin embargo, investigaciones indican que es posible mejorar la fuerza a partir de disminuciones en variables a composición corporal. Es así como Perry, Van Guilder, Kauffman, \& Hossain, (2020) llevaron a cabo un programa de restricción calórica en un grupo de adultos mayores $(n=36)$ obesos durante 12 semanas, encontrando efectos positivos sobre la medida de fuerza evaluada mediante prensión manual, concluyendo que disminuciones de porcentaje de tejido adiposo e incrementos de masa muscular favorecen esta cualidad. A diferencia de nuestro estudio, además de IMC, el resto de las mediciones de composición corporal fueron realizadas mediante bioimpedancia. En la misma línea, Farrell et al., (2019), 
realizaron un estudio en el cual se analizó la relación entre capacidad funcional, evaluada mediante el Functional Movement Screen, y algunos marcadores de salud, reportándose correlaciones negativas bajas y significativas entre los resultados del test e IMC $(\mathrm{r}=-0.366$ $-\mathrm{p}<0.00)$.

Como principal limitación de nuestro estudio, es que el trabajo se presenta en una característica transversal lo cual no ofrece indicativos para considerar las adaptaciones morfofisiológicas del estado de acondicionamiento físico frente a las actividades propuestas en el taller. Sumado a esto, el tamaño de la muestra, así como el tipo de muestreo son considerados como tal. Por otro lado, consideramos que la fortaleza del estudio es el control de los criterios de inclusión para considerar solamente las mujeres que no presentaban condiciones patológicas que pudiesen interferir en el resultado de los test, la homogeneidad de las características antropométricas de los diferentes grupos, reduciendo las interferencias de esas variables en las respuestas del test y en la originalidad de la propuesta para la población chilena.

\section{Conclusión}

Los resultados de esta investigación demuestran que tanto la edad como el IMC son predictores de laVM en mujeres mayores y que el deterioro funcional, reflejado en la disminución de los resultados de la capacidad de desplazamiento, disminuye de forma ostensible con el paso de los años, recomendándose intervenciones específicas que lo aminoren. Por otro lado, no se observó una relación significativa de tendencia lineal entre disminución de los resultados de la capacidad de desplazamiento y la fuerza máxima de tren superior y también que la PM no presentó disminución significativa en función de edad de la población de mujeres mayores chilenas que participan de programa de actividad física. Se recomienda la realización de estudios que puedan corroborar o contestar las informaciones presentadas en el presente estudio, la posibilidad de generar indicadores y test que puedan contribuir para mejores evaluaciones del acondicionamiento físico y los impactos del envejecimiento en la población mayor.

\section{Agradecimientos}

Agradecimientos especiales al Instituto Nacional de Deportes de Nuble región por facilitar el acceso a la muestra de adultos mayores.

\section{Referencias}

Adell, E., Wehmhörner, S., \& Rydwik, E. (2013). The testretest reliability of 10 meters maximal walking speed in older people living in a residential care unit. Journal of Geriatric Physical Therapy, 36(2), 74-77.https://doi.org/ 10.1519/JPT.0b013e318264b8ed

Alcañiz, R. N., \& González-Moro, I. M. (2020).Valoración del grado de deterioro funcional y fragilidad en adultos mayores activos. Retos, 38, 576-581. https://doi.org/ https://doi.org/10.47197/retos.v38i38.78252

Alzina, R. B. (1987). Introducción a la estadística aplicada a la investigación educativa: un enfoque informático con los paquetes BMDP y SPSSX. (P. Promociones y Publicaciones Universitarias, Ed.).

Arnold, M., Herrera, F., Massad, C., \& Thumala, D. (2018). Quinta encuesta nacional de inclusión y exclusión social de las personas mayores en Chile: Opiniones de la población chilena respecto al envejecimiento poblacional.

Bellomo, R. G., Iodice, P., Maffulli, N., Maghradze,T., Coco, V., \& Saggini, R. (2013). Muscle strength and balance training in sarcopenic elderly: A pilot study with randomized controlled trial. European Journal of Inflammation, 11(1), 193-201. https://doi.org/10.1177/ $1721727 X 1301100118$

Bowden, K. A., Pickles, S., Sprung, V. S., Kemp, G. J., Alam, U., Moore, D. R., , . Cuthbertson,D. J. (2019). Reduced physical activity in young and older adults: metabolic and musculoskeletal implications. Therapeutic Advances in Vaccines, 10,1-15. https://doi.org/10.1177/https

Casas-Herrero, A., Anton-Rodrigo, I., Zambom-Ferraresi, F., Sáez De Asteasu, M. L., Martinez-Velilla, N., Elexpuru-Estomba, J., ... Izquierdo, M. (2019). Effect of a multicomponent exercise programme (VIVIFRAIL) on functional capacity in frail community elders with cognitive decline: Study protocol for a randomized multicentre control trial. Trials, 20(1), 1-12. https:// doi.org/10.1186/s13063-019-3426-0

Chou, M.Y., Nishita, Y., Nakagawa, T., Tange, C., Tomida, M., Shimokata, H., ... Arai, H. (2019). Role of gait speed and grip strength in predicting 10-year cognitive decline among community-dwelling older people. BMC Geriatrics, 19(1), 1-11. https://doi.org/10.1186/ s12877-019-1199-7

Cruz-Jentoft, A. J., Bahat, G., Bauer, J., Boirie,Y., Bruyère, O., Cederholm, T., ... Schols, J. (2019). Sarcopenia: Revised European consensus on definition and diagnosis. Age and Ageing, 48, 16-31. https://doi.org/10.1093/ ageing/afy169

De Labra, C., Guimaraes-Pinheiro, C., Maseda, A., Loren- 
zo, T., \& Millán-Calenti, J. C. (2015). Effects of physical exercise interventions in frail older adults: A systematic review of randomized controlled trials Physical functioning, physical health and activity. BMC Geriatrics, 15(1). https://doi.org/10.1186/s12877-015-0155-4

Drey, M., Sieber, C. C., Bertsch, T., Bauer, J. M., Schmidmaier, R., \& The FiAT intervention group. (2016). Osteosarcopenia is more than sarcopenia and osteopenia alone. Aging Clinical and Experimental Research, 28(5), 895-899.https://doi.org/10.1007/s40520-0150494-1

Farrell, S. W., Pavlovic, A., Barlow, C. E., Leonard, D., DeFina, J. R., Willis, B. L., ... Haskell, W. L. (2019). Functional Movement Screening Performance and Association With Key Health Markers in Older Adults. Journal of Strength and Conditioning Research, 1(29), 1-7. https://doi.org/10.1519/JSC.0000000000003273

Fragala, M. S., Cadore, E. L., Dorgo, S., Izquierdo, M., Kraemer,W. J., Peterson, M. D., \& Ryan, E. D. (2019). Resistance Training for Older Adults: Position Statement From the National Strength and Conditioning Association. Journal of Strength and Conditioning Research, 33(8), 2019 2052. JSC.0000000000003230

Fragala, M. S., Clark, M. H., Walsh, S. J., Kleppinger, A., Judge, J. O., Kuchel, G. A., \& Kenny, A. M. (2012). Gender differences in anthropometric predictors of physical performance in older adults. Gender Medicine, 9(6), 445-456. https://doi.org/10.1016/ j.genm.2012.10.004

Garber, C. E., Blissmer, B., Deschenes, M. R., Franklin, B. A., Lamonte, M. J., Lee, I. M., ... Swain, D. P. (2011). Quantity and quality of exercise for developing and maintaining cardiorespiratory, musculoskeletal, and neuromotor fitness in apparently healthy adults: Guidance for prescribing exercise. Medicine and Science in Sports and Exercise, 43(7), 1334-1359. https: / /doi.org/10.1249/ MSS.0b013e318213fefb

Gonzalez, M., Gates, D. H., \& Rosenblatt, N. J. (2020). The impact of obesity on gait stability in older adults. Journal of Biomechanics, 100, 109585. https: / / doi.org/10.1016/ j.jbiomech.2019.109585

Guralnik, J. M., Ferrucci, L., Pieper, C. F., Leveille, S. G., Markides, K. S., Ostir, G. V., ... Wallace, R. B. (2000). Lower extremity function and subsequent disability: Consistency across studies, predictive models, and value of gait speed alone compared with the short physical performance battery. Journals of Gerontology - Series A Biological Sciences and Medical Sciences, 55(4), 221-231. https: / / doi.org/10.1093/gerona/55.4.M221
Heiland, E. G., Welmer, A. K., Wang, R., Santoni, G., Angleman, S., Fratiglioni, L., \& Qiu, C. (2016). Association of mobility limitations with incident disability among older adults: a population-based study. Age and Ageing, 45(6), 812-819. https://doi.org/10.1093/ ageing/afw076

Jadczak, A. D., Makwana, N., Luscombe-Marsh, N., Visvanathan, R., \& Schultz, T. J. (2018). Effectiveness of exercise interventions on physical function in community-dwelling frail older people: an umbrella review of systematic reviews. JBI Database of Systematic Reviews and Implementation Reports, 16(3), 752-775. https: / /doi.org/10.11124/JBISRIR-2017-003551

Kendrick, D., Kumar, A., Delbaere, K., Zijlstra, G. A. R., Carpenter, H., Iliffe, S., . . Morris, R. (2014). Exercise for reducing fear of falling in older people living in the community: Cochrane systematic review and MetaAnalysis. Age and Ageing, 45(3), 345-352. https:// doi.org/10.1093/ageing/afw036

Kuki, A., Tanaka, K., Kushiyama, A., Tanaka, Y., Motonishi, S., Sugano, Y., ... Ozawa, T. (2019). Association of gait speed and grip strength with risk of cardiovascular events in patients on haemodialysis: A prospective study. BMC Nephrology, 20(1), 1-10. https://doi.org/10.1186/ s12882-019-1370-6

Lavedán Santamaría, A., Jürschik Giménez, P., Botigué Satorra, T., Nuin Orrio, C., \& Viladrosa Montoy, M. (2015). Prevalencia y factores asociados a caídas en adultos mayores que viven en la comunidad. Atencion Primaria, 47(6), 367-375. https://doi.org/10.1016/ j.aprim.2014.07.012

Mancilla S, E., RamosF, S., \& Morales B, P.(2016).Association between handgrip strength and functional performance in Chilean older people. Revista Medica de Chile, 144(5), 598-603. https://doi.org/10.4067/S003498872016000500007

Mathiowetz, V. (2002). Comparison of Rolyan and Jamar dynamometers for measuring grip strength. Occupational Therapy International, 9(3), 201-209. https://doi.org/ 10.1002/oti.165

Mendonca, G. V., Pezarat-Correia, P., Vaz, J. R., Silva, L., Almeida, I. D., \& Heffernan, K. S. (2016). Impact of Exercise Training on Physiological Measures of Physical Fitness in the Elderly. Current Aging Science, 9(4), 240 259. 1874609809666160426120600

Muehlbauer, T., Besemer, C., Wehrle, A., Gollhofer, A., \& Granacher, U. (2012). Relationship between strength, power and balance performance in seniors. Gerontology, 58(6), 504-512.https://doi.org/10.1159/000341614 
Norton, K., \& Olds, T. (1996). Anthropometrica: A Texbook of Body Measurement for Sports and Health Education (First edit). Sydney: CBS Publishers \& Distributors.

Novaes, R. D., Miranda, A. S., \& Dourado, V. Z. (2011). Velocidade usual da marcha em brasileiros de meia idade e idosos. Revista Brasileira de Fisioterapia, 15(2), 117-122. https: / doi.org/10.1590/S1413-35552011000200006

OMS. (2015). OMS | Datos interesantes acerca del envejecimiento. WHO. Retrieved from https:// www.who.int/ageing/about/facts/es/

OMS. (2018). Caídas, Datos y Cifras. Retrieved January 11,2020, from https: / /www.who.int/es/news-room/ fact-sheets/detail/falls

Perry, C. A., Van Guilder, G. P., Kauffman, A., \& Hossain, M. (2020). A calorie-restricted DASH diet reduces body fat and maintains muscle strength in obese older adults. Nutrients, 12(1), 1-14. https://doi.org/10.3390/ nu12010102

Poblete-Valderrama, F., Flores-Rivera, C., PetermannRocha, F., Leiva, A. M., Martínez-Sanguinetti, M. A., Troncoso, C., ... Celis-Morales, C. (2019). Actividad física y tiempo sedente se asocian a sospecha de deterioro cognitivo en población adulta mayor chilena. Revista Medica de Chile, 147, 1247-1255.

Pozo-Cruz, J. Del, Magaña, M., Ballesteros, M., Porras, M., Rodríguez, E., Navas, P., \& López-Lluch, G. (2013). Medicina del deporte. Revista Andaluza de Medicina Del Deporte, 6(2), 57-65. https://doi.org/10.36104/ amc.2018.1400

Rijk, J. M., Roos, P. R. K. M., Deckx, L., Van den Akker, M., \& Buntinx, F. (2016). Prognostic value of handgrip strength in people aged 60 years and older: A systematic review and meta-analysis. Geriatrics and Gerontology International, 16(1), 5-20. https://doi.org/10.1111/ ggi. 12508

Roberts, H. C., Syddall, H. E., Sparkes, J., Ritchie, J., Butchart, J., Kerr, A., ... Sayer, A. A. (2014). Grip strength and its determinants among older people in different healthcare settings. Age and Ageing, 43(2), 241246. https: //doi.org/10.1093/ageing/aft118

Rydwik, E., Bergland, A., Forsén, L., \& Frändin, K. (2012). Investigation into the reliability and validity of the measurement of elderly people's clinical walking speed: A systematic review. Physiotherapy Theory and Practice, 28(3), 238-256. https://doi.org/10.3109/ 09593985.2011.601804

Sallinen, J., Stenholm, S., Rantanen, T., Heliövaara, M., Sainio, P., \& Koskinen, S. (2010). Hand-Grip Strength Cut-Points to Screen Older Persons at Risk for Mobility Limitation. Bone, 23(1), 1-7.https://doi.org/10.1038/ jid.2014.371

Sherrington, C., Fairhall, N. J., Wallbank, G. K., Tiedemann, A., Michaleff,Z. A., Howard, K., ... Lamb, S. E. (2019). Exercise for preventing falls in older people living in the community. Cochrane Database of Systematic Reviews, 2019(1), 1-8. https://doi.org/10.1002/ 14651858.CD012424.pub2

Siemonsma, P. C., Blom, J. W., Hofstetter, H., Van Hespen, A.T. H., Gussekloo, J., Drewes,Y. M., \& Van Meeteren, N. L. U. (2018). The effectiveness of functional task exercise and physical therapy as prevention of functional decline in community dwelling older people with complex health problems. BMC Geriatrics, 18(1), 1-8. https://doi.org/10.1186/s12877-018-0859-3

Studenski, S., Perera, S., Patel, K., Rosano, C., Faulkner, K., \& Inzitari, M. (2011). Gait Speed and Dismobility in Older Adults. JAMA Internal Medicine, 305(1), 50-58. https: / /doi.org/10.1016/j.apmr.2015.05.017

Valenzuela, P.L., Castillo-García,A., Morales, J. S., Izquierdo, M., Serra-Rexach, J. A., Santos-Lozano, A., \& Lucia, A. (2019). Physical exercise in the oldest old. Comprehensive Physiology, 9(4), 1281-1304.https:/ /doi.org/10.1002/ cphy.c190002

Van Houwelingen, A. H., Cameron, I. D., Gussekloo, J., Putter, H., Kurrle, S., De Craen, A. J. M., ... Blom, J. W. (2014). Disability transitions in the oldest old in the general population. The Leiden 85-plus study. Age, 36(1), 483-493. https: / /doi.org/10.1007/s11357-013-95743

Villalobos, P. (2019). Panorama de la dependencia en Chile: avances y desafíos. Revista Médica de Chile, 147(1), 83-90. https: / /doi.org/10.4067/s0034-98872019000100083

Villarreal-Angeles, M. A., Moncada-Jimenez, J., \& RuizJuan, F.(2020). Mejora de variablespsicológicas en Adultos Mayores mediante Pilates (Improvement of psychological variables in Older Adults through Pilates). Retos, 40, 47-52. https://doi.org/10.47197/ retos.v1i40.74307

Yang, S., Li, T., Yang, H., Wang, J., Liu, M., Wang, S., ... Jiang, B. (2020). Association between muscle strength and health-related quality of life in a Chinese rural elderly population: a cross-sectional study. BMJ Open, 10(1), e026560. https://doi.org/10.1136/bmjopen-2018026560

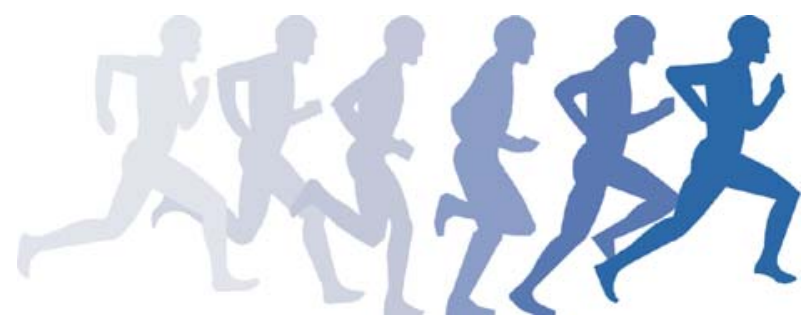

